\section{Age, smoking, hypertension, and aortic aneurysm: Interactions and risks}

\section{Seriki SA* and Otoikhila OC}

Department of Human Physiology, College of Medical Sciences, Edo State University, Uzairue, Nigeria

\section{Abstract}

Background/Aim: Aortic aneurysm is the bulging of a weakened portion of the aorta. The aorta is the major blood vessel that feeds blood (carrying oxygen, nutrients and water) to the tissues of the body. When a portion of the wall of the aorta becomes weak, blood pushing against the vessel wall can cause it to bulge like a balloon (aneurysm) leading to aortic dissection (a tear in the wall of the aorta that can cause life-threatening bleeding or sudden death). Blood pressure is the force arterial blood exerts on the wall of the artery. When this pressure is consistently high above $140 / 100 \mathrm{mmHg}$ it is referred to as hypertension. As an individual gets older over time, physiological functions of the body depreciate leading to some abnormalities. Smoking is the consumption of tobacco mostly by inhalation of the smoke that is produced from burning the tobacco. This review article examines the close interactions between age, smoking, hypertension and aortic aneurysm, with a view to understanding mechanisms by which these factors predispose a patient to an aortic aneurism. It is also to observe if these factors interfere with treatment and recovery from aneurysms.

Conclusion: After careful review, it is observed that age and smoking are risk factors for hypertension, and together with hypertension, the three factors predispose an individual to high risk for aortic aneurysm.

\section{More Information}

*Address for Correspondence: Seriki Samuel Adinoyi, Department of Human Physiology, College of Medical Sciences, Edo State University, Uzairue, Nigeria, Email: seriki.adinoyi@gmail.com

Submitted: December 15, 2021 Approved: January 05, 2022 Published: January 06, 2022

How to cite this article: Seriki SA, Otoikhila OC. Age, smoking, hypertension, and aortic aneurysm: Interactions and risks. J Cardiol Cardiovasc Med. 2022; 7: 001-005.

DOI: 10.29328/journal.jccm.1001123

Copyright License: (c) 2022 Seriki SA, et al. This is an open access article distributed under the Creative Commons Attribution License, which permits unrestricted use, distribution, and reproduction in any medium, provided the original work is properly cited.

Keywords: Hypertension; Weak aorta; Aortic dissection; High blood pressure; Smoking

Check for updates

OPEN ACCESS

\section{Introduction}

An aortic aneurysm is an enlargement of the aorta to greater than 1.5 times normal size. Though common with abdominal aorta, aortic aneurysm could also occur in the thoracic aorta; ascending aorta, aortic arch, or descending aorta.

Aortic aneurysms often grow slowly and usually without symptoms, making them difficult to detect. They cause weakness in the wall of the aorta and may even lead to aortic rupture. Some aneurysms may however never rupture throughout their lifetime; many start small and remain small, although some expand over time. The rate of growth of an aneurysm may depend on the level of the weakness of the aortic wall. When rupture occurs, massive internal bleeding results, leading to a medical emergency, and unless timely treatment is given it could lead to shock and death. Except when it ruptures, the aortic aneurysm may not present any serious symptom. Occasionally, there may be abdominal, back, or leg pain/numbness due to compression of nerve roots. A common sign is a hoarse voice from the stretching of the left recurrent laryngeal nerve, a branch of the vagus nerve that winds around the aortic arch to supply the muscles of the larynx $[1,2,3]$.

Depending on the cause, size and growth rate of aortic aneurysm, treatment may vary from careful monitoring to emergency surgery [4].

Factors that may contribute to an aneurysm's development include:

- Hardening of the arteries (atherosclerosis). As plaque builds up on the artery walls, they become less flexible, and additional pressure can cause them to weaken and bulge. High blood pressure and high cholesterol are risk factors for the hardening of the arteries. These are common in older people. These risk factors predispose individuals to all types of aneurysms.

- Genetic conditions. Aortic aneurysms are uncommon in younger persons, but when they occur they often have a genetic cause. Patients born with Marfan syndrome, a genetic condition that affects the connective tissue 
in the body, are particularly at risk of a thoracic aortic aneurysm because they may have weakness in the aortic wall.

Marfan syndrome generally causes distinct physical traits, including tall stature, very long arms, a deformed breastbone and eye problems.

Other genetic disorders can cause an aortic aneurysm, including vascular Ehlers-Danlos, Loeys-Dietz and Turner syndromes. Vascular Ehlers-Danlos syndrome causes the skin, joints and connective tissue to be fragile and makes the skin stretch easily.

\section{Classification}

Aortic aneurysms may be classified by their location on the aorta.

*Thoracic Aortic Aneurysms (TAA) are found within the chest; these are further classified as ascending aortic aneurysm, which presents bulging of the ascending aorta; aortic arch aneurysm, which presents bulging at the aortic arch; or descending aortic aneurysms, which is when the descending aorta bulges at the thoracic region (Figure 1).

*Abdominal aortic aneurysms (AAA) involve the segment of the aorta within the abdominal cavity/region. They are the most common forms of aortic aneurysm. Abdominal aortic aneurysms (AAA) are about $3.4 \mathrm{~cm}$. One reason AAA is common is that elastin, the principal load-bearing protein present in the wall of the aorta, is reduced in the abdominal aorta as compared to the thoracic aorta. Another reason is that the abdominal aorta does not possess vasa vasorum, the nutrient-supplying blood vessels within the wall of the aorta. Most AAA are true aneurysms that involve all three layers of the blood vessels (tunica intima, tunica media and tunica adventitia). The rupture of an AAA is dependent on its diameter; once the aneurysm reaches about $5 \mathrm{~cm}$, the yearly risk of rupture may exceed the risks of surgical repair for an

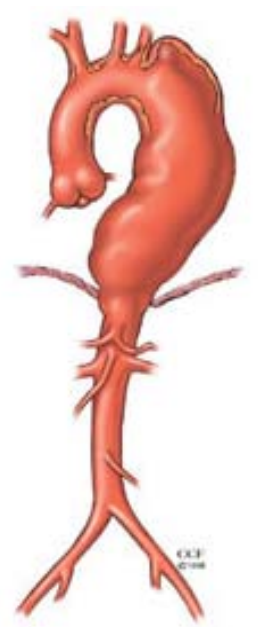

Figure 1: Image showing typical example of thoracic aortic aneurysm occurring at the descending aorta. average-risk patient. Rupture risk is also related to shape; long aneurysms are considered less rupture-prone than shorter, bulbous aneurysms; the latter having more wall tension in a particular location in the aneurysm wall [5].

Before rupture, an AAA maypresentas a large, pulsatile mass above the umbilicus. A bruit may be heard from the turbulent flow in the aneurysm. Unfortunately, however, rupture may be the first hint of AAA. Once an aneurysm has ruptured, it presents with classic symptoms of abdominal pain which is severe, persistent, and radiating to the back [6]. Only $10 \%-25 \%$ of patients survive rupture due to large pre-and postoperative mortality.

The most common location of arterial aneurysm formation is the abdominal aorta, specifically, the segment of the abdominal aorta below the kidneys. An abdominal aneurysm located below the kidneys is called an infrarenal aneurysm. When an aneurysm is located above the kidneys it is described as suprarenal.

*Thoracoabdominal aortic aneurysms involve the aorta at the border between the thorax and the abdomen and have components of both thoracic and abdominal aortic aneurysms [7].

An aneurysm can be characterized by its location, shape, and cause.

The shape of an aneurysm is described as being fusiform or saccular which helps to identify a true aneurysm. The more common fusiform-shaped aneurysm bulges or balloons out on all sides of the aorta. A saccular-shaped aneurysm bulges or balloons out only on one side [8].

A pseudoaneurysm, or false aneurysm, is an enlargement of only the outer layer of the blood vessel wall. A false aneurysm may be the result of prior surgery or trauma. Sometimes, a tear can occur on the inside layer of the vessel resulting in blood filling in between the layers of the blood vessel wall creating a pseudo-aneurysm [8].

The aorta is under constant pressure as blood is ejected from the heart. With each heartbeat, the walls of the aorta distend (expand) and then recoil (spring back), exerting continual pressure or stress on the already weakened aneurysm wall. Therefore, there is a potential for rupture (bursting) or dissection (separation of the layers of the aortic wall) of the aorta, which may cause life-threatening hemorrhage (uncontrolled bleeding) and, potentially, death. The larger the aneurysm becomes, the greater the risk of rupture [8].

\section{Risk factors for aortic aneurysm}

Age, smoking and hypertension have been implicated in the development of an aneurysm. These factors tend to greatly increase the risk of developing it. 


\section{Age and aortic aneurysm}

Thoracic aortic aneurysms occur most often in people age 65 and older. Experts have said that at that age the aorta may have lost its capacity of elasticity. Therefore it is not able to dilate. The buildup of blood within the aorta predisposes an individual to an aortic aneurysm.

The build-up of plaques in the arteries such as fat and other substances that can damage the lining of a blood vessel increases the risk of an aneurysm. This has been observed to be a more common risk in older persons. Abdominal aortic aneurysm, for instance, increases with age, with an average age of 65-70 at the time of diagnosis. It has been attributed to atherosclerosis, though other factors may be involved in their formation [7].

Since AAA is common in the elderly, the risks of postoperative complications should become a challenge. Frailty is also common in elderly people, and the prevalence of frailty increases with coexisting cardiovascular diseases [9].

Doxycycline, abroadinhibitor of matrix-metalloproteinases has been observed by researchers to exhibit a potential to slow down aortic aneurysm growth in a previous study [10], this has however been criticized by another research work which observed that doxycycline has no significant effect on the growth of aortic aneurysm.

\section{Smoking and aortic aneurysm}

Tobacco use, either by smoking or sniffing is a strong risk factor for the development of an aortic aneurysm, with current smokers standing 5 -fold increase risk and former smokers standing 2-fold increase risk compared to never smokers.

In addition, there is a strong dose-response relation between the increasing number of cigarettes per day and pack-years and the increasing risk of abdominal aortic aneurysms. This is in addition to a linear inverse association between increasing duration of smoking cessation and risk of abdominal aortic aneurysms, with risk approaching that of never smokers at 25 years of smoking cessation. This means that smokers that have ceased smoking for up to 25 years stand almost equal risk as someone that never smoked.

Smokers oftentimes have a less healthy lifestyle than non-smokers with lower physical activity, more abdominal adiposity, and unhealthier diets [11].

Abdominal aortic aneurysms are characterized by loss of normal medial arterial structure (tunica media) and the nearcomplete absence of normal lamellar elastin matrix [12].

The breakdown of the elastin and collagen of the arterial media is mediated by matrix metalloproteinases released by macrophages and is also related to chronic inflammatory infiltration and loss or dysfunction of parenchymal cells central to matrix deposition and repair. Smoking may activate tissue plasminogen activator which induces the production of matrix metalloproteinases by macrophages and has also been shown to disrupt collagen synthesis $[13,14]$.

A rat study had shown that nicotine administration weakens the vascular wall, increases gelatinolytic activity and promoted the destruction of elastin and collagen in the abdominal aorta, leading to up-regulation of matrix metalloproteinase-12 expression [15]. Smoking wreaks its havoc by weakening the aortic walls, increasing the possibility not only of developing an aneurysm but also of causing a dissection.

Both tobacco smoke and benzopyrene, a compound found in cigarette smoke, increased aortic muscular cell apoptosis and aortic macrophage infiltration and expression of matrix metalloproteinases-2, 9 and 12 and nuclear factor- $\mathrm{\kappa B}$ and increased formation of abdominal aortic aneurysms in angiotensin-2 induced hypertension [16,17].

\section{Hypertension and aortic aneurysm}

Increased blood pressure damages the blood vessels in the body, raising the chances of developing an aneurysm. It is believed that hypertension weakens the aneurysmal wall by directly increasing mechanical stresses.

Previous studies have revealed that there is a strong relationship between hypertension and Aortic aneurysm, with about $66 \%$ increased risk of developing aortic aneurysm in patients with hypertension compared to non-hypertensive patients [18]

It is also striking to note that Diastolic Pressure (DP) increase compounds the risk of aneurysm compared to Systolic Pressure (SP) increase, with about $20 \mathrm{mmHg}$ increase in SP and a $10 \mathrm{mmHg}$ increase in DP associated causing $14 \%$ and $28 \%$ increase in the risk of developing Aortic Aneurysm respectively [18]

High SP and low DP are associated with arterial stiffness which indicates strong protein fiber [19], which is protective against a weakening of aortic tissues and could be responsible for the stronger association observed between DP compared to SP [20].

It has been observed that activation of the local ReninAngiotensin System (RAS) by systemic hypertension can cause vascular inflammation and remodeling, and may contribute to aneurysmal rupture [21].

Another striking incites, though without any obvious scientific reason is the fact that aortic aneurysm is five-fold present in men than in women [22]. The possible explanation could be a higher prevalence of other risk factors more common with men, such as smoking and alcoholism [23], which would increase the absolute risk of hypertension among men, and any additional adverse relative effect of 
hypertension might therefore be less among women because of their healthier lifestyle.

The association between hypertension and AAA could potentially be confounded by other risk factors because hypertension is more common among persons with overweight and obesity, less physical activity, who smoke and who have unhealthy diets.

\section{Prevention}

The risk of aneurysm enlargement may be diminished with attention to the patient's blood pressure, smoking and cholesterol levels. Blood pressure should be kept within the normal range of 100 to $139 \mathrm{mmHg}$ systolic and 70 to $89 \mathrm{mmHg}$ diastolic values. Patients are also advised to quit smoking, as that will drastically reduce the risk. There have been proposals to introduce ultrasound scans as a screening tool for those most at risk: men over the age of $65[24,25]$.

The tetracycline antibiotic doxycycline is currently being investigated for use as a potential drug in the prevention of aortic aneurysm due to its metalloproteinase inhibitor and collagen stabilizing properties.

Anacetrapib is a cholesteryl ester transfer protein inhibitor that raises high-density lipoprotein (HDL) cholesterol and reduces low-density lipoprotein (LDL) cholesterol. Anacetrapib reduces the progression of atherosclerosis, mainly by reducing non-HDL-cholesterol, improves lesion stability and adds to the beneficial effects of atorvastatin [26]. Elevating the amount of HDL cholesterol in the abdominal area of the aortic artery in mice both reduced the size of aneurysms that had already grown and prevented abdominal aortic aneurysms from forming at all. In short, raising HDL cholesterol is beneficial because it induces programmed cell death. The walls of a failing aorta are replaced and strengthened. New lesions should not form at all when using this drug [27].

\section{Discussion}

\section{Smoking}

Recent research has revealed that there is a 5 -fold and 2 -fold increase in the risk of abdominal aortic aneurysms among current and former smokers compared to never smokers, respectively. A dose-response relationship was also observed between the increasing number of cigarettes smoked per day and pack-years smoked and the risk of abdominal aortic aneurysm, while there was a reduced risk with increasing duration of smoking cessation with a risk similar to that of never smokers at about 25 years of smoking cessation. These together with supportive experimental data provide strong evidence for a causal relationship between smoking and abdominal aortic aneurysms. Smokers are therefore advised on the risk of vulnerability to aortic aneurism, and should therefore quit. The findings provide further support for interventions and policies to curb the global tobacco epidemic.

\section{Hypertension}

Hypertension too has been observed as predisposing individuals to aortic aneurysms as abnormally increased blood pressure vessels exert serious impact on the wall of the aorta and its tissues. It is even more striking to observe that the impact of the Diastolic Pressure is even higher than the Systolic Pressure in exposing individuals to aortic aneurysm.

\section{Age}

Thoracic aortic aneurysms occur most often in people age 65 and older. Experts have said that at that age the aorta may have lost its capacity of elasticity. Therefore it is not able to dilate. The buildup of blood within the aorta predisposes an individual to an aortic aneurysm.

\section{Conclusion}

Given observed interactions between smoking, hypertension, age, and aortic aneurysm, in which the factors pose a risk to aneurysm, the aging population should be regularly screened for the aneurysm. Also, smokers are advised to quit smoking to prevent predisposition, while the blood pressure of hypertensive patients should be kept under control below $140 / 90 \mathrm{mmHg}$. With the poor rate of survival of patients with aneurysms, attention should focus more on screening and prevention than treatment.

\section{References}

1. Johnston KW, Rutherford RB, Tilson MD, Shah DM, Hollier L, et al. Suggested standards for reporting on arterial aneurysms. Subcommittee on Reporting Standards for Arterial Aneurysms, Ad Hoc Committee on Reporting Standards, Society for Vascular Surgery and North American Chapter, International Society for Cardiovascular Surgery. J Vasc Surg. 1991; 13: 452-458.

PubMed: https://pubmed.ncbi.nlm.nih.gov/1999868/

2. Kent KC. Abdominal Aortic Aneurysms. N Engl J Med. 2014; 371 2101-2108.

PubMed: https://pubmed.ncbi.nlm.nih.gov/25427112/

3. Upchurch GR, Schaub TA. Abdominal aortic aneurysm. Am Fam Physician. 2006; 73: 1198-1204.

PubMed: https://pubmed.ncbi.nlm.nih.gov/16623206/

4. GBD 2013 Mortality and Causes of Death, Collaborators.. Global, regional, and national age-sex specific all-cause and cause-specific mortality for 240 causes of death, 1990-2013: a systematic analysis for the Global Burden of Disease Study 2013. Lancet. 2015; 385: 117-171. PubMed: https://pubmed.ncbi.nlm.nih.gov/25530442/

5. Abdominal Aortic Aneurysms. The Lecturio Medical Concept Library. 16 October 2020. 2021.

6. Vu K, Kaitoukov Y, Morin-Roy F, Kauffmann C, Tang A, et al. Rupture signs on computed tomography, treatment, and outcome of abdominal aortic aneurysms. Insights Imaging. 2014; 5: 281-293. PubMed: https://pubmed.ncbi.nlm.nih.gov/24789068/

7. Mathur A, Mohan V, Ameta D, Gaurav B, Haranahalli P. Aortic aneurysm. J TransI Inter Med. 2016; 4: 35-41.

PubMed: https://www.ncbi.nlm.nih.gov/pmc/articles/PMC5290913/

8. Freiberg MS, Arnold AM, Newman AB, Edwards MS, Kraemer KL, et al. Abdominal aortic aneurysms, increasing infrarenal aortic diameter, and risk of total mortality and incident cardiovascular disease events. Circulation. 2008; 117: 1010-1017.

PubMed: https://pubmed.ncbi.nlm.nih.gov/18268154/ 
9. Takeuchi H, Uchida HA, Kakio Y, Okuyama Y, Okuyama M, et al. The Prevalence of Frailty and its Associated Factors in Japanese Hemodialysis Patients. Aging Dis. 2018; 9: 192-207. PubMed: https://pubmed.ncbi.nlm.nih.gov/29896410/

10. Howard DPJ, Banerjee A, Fairhead JF, Handa A, Silver LE, et al. Agespecific incidence, risk factors and outcome of acute abdominal aortic aneurysms in a defined population Br J Surg. 2015; 102: 907-915. PubMed: https://pubmed.ncbi.nlm.nih.gov/25955556/

11. Stackelberg O, Bjorck M, Larsson SC, Orsini N, Wolk A. Fruit and vegetable consumption with risk of abdominal aortic aneurysm. Circulation. 2013; 128: 795-802.

PubMed: https://pubmed.ncbi.nlm.nih.gov/23960255/

12. Norman PE, Curci JA. Understanding the effects of tobacco smoke on the pathogenesis of aortic aneurysm. Arterioscler. Thromb Vasc Biol. 2013; 33: 1473-1477. PubMed: https://pubmed.ncbi.nlm.nih. gov/23685557/

13. Lindholt JS, Jorgensen B, Shi GP, Henneberg EW. Relationships between activators and inhibitors of plasminogen, and the progression of small abdominal aortic aneurysms. Eur J Vasc Endovasc Surg. 2003; 25: 546-551. PubMed: https://pubmed.ncbi.nlm.nih.gov/12787697/

14. Wilson KA, Lindholt JS, Hoskins PR, Heickendorff L, Vammen S, et al. The relationship between abdominal aortic aneurysm distensibility and serum markers of elastin and collagen metabolism. Eur $\mathrm{J}$ Vasc Endovasc Surg. 2001; 21: 175-178.

PubMed: https://pubmed.ncbi.nlm.nih.gov/11237793/

15. Kugo H, Zaima N, Tanaka H, Urano T, Unno N, et al. The effects of nicotine administration on the pathophysiology of rat aortic wall. Biotech. Histochem. 2017; 92: 141-148.

PubMed: https://pubmed.ncbi.nlm.nih.gov/28296545/

16. Ji K, Zhang $Y$, Jiang $F$, Qian L, Guo $H$, et al. Exploration of the mechanisms by which 3,4-benzopyrene promotes angiotensin IIinduced abdominal aortic aneurysm formation in mice. J Vasc Surg. 2014; 59: 492-499.

PubMed: https://pubmed.ncbi.nlm.nih.gov/23676189/

17. Stolle K, Berges A, Lietz M, Lebrun S, Wallerath T. Cigarette smoke enhances abdominal aortic aneurysm formation in angiotensin II-treated apolipoprotein E-deficient mice. Toxicol Lett. 2010; 199: 403-409. PubMed: https://pubmed.ncbi.nlm.nih.gov/20937366/

18. Elsa K, Makoto H, Han P, Dagfinn A. Blood Pressure, hypertension and the risk of abdominal aortic aneurysms: a systemic review and metaanalysis of cohot studies. European J Epidemiol. 2019; 34: 547-555. PubMed: https://pubmed.ncbi.nlm.nih.gov/30903463/

19. Samuel SA, Francis AO, Anthony OO. Role of the Kidneys in the Regulation of Intra- and Extra-Renal Blood Pressure. Ann Clin Hypertens. 2018; 2: 048-058.

https://www.heighpubs.org/hjch/ach-aid1011.php

20. Rapsomaniki E, Timmis A, George J, Pujades-Rodriguez M, Shah AD, et al. Blood pressure and incidence of twelve cardiovascular diseases: lifetime risks, healthy life-years lost, and age-specific associations in 1.25 million people. Lancet. 2014; 383: 1899-1911. PubMed: https://pubmed.ncbi.nlm.nih.gov/24881994/

21. Ohkuma H, Suzuki S, Fujita S, Nakamura W. Role of decreased expression of the local renin-agiotensin system in the etiology of cerebral aneurysms. Circulation. 2003; 108: 785-787. PubMed: https://pubmed.ncbi.nlm.nih.gov/12912805/

22. DeRubertis BG, Trocciola SM, Ryer EJ, Pieracci FM, McKinsey JF, et al. Abdominal aortic aneurysm in women: prevalence, risk factors, and implications for screening. J Vasc Surg. 2007; 46: 630-635. PubMed: https://pubmed.ncbi.nlm.nih.gov/17903646/

23. Guindon GE, Boisclair D. Past, current and future trends in tobacco use: HNP discussion paper. Washington: The World Bank. 2018. http://escholarship.org/uc/item/4q57d5vp

24. Routine screening in the management of AAA, UK Department of Health study Report Archived. 2007.

25. Mortality results for randomised controlled trial of early elective surgery or ultrasonographic surveillance for small abdominal aortic aneurysms. Lancet. 352: 1649-1655.

PubMed: https://pubmed.ncbi.nlm.nih.gov/9853436/

26. Bloomfield D, Carlson GL, Sapre A, Tribble D, McKenney JM, et al. Efficacy and safety of the cholesteryl ester transfer protein inhibitor anacetrapib as monotherapy and coadministered with atorvastatin in dyslipidemic patients. Am Heart J. 2009; 157: 352-360. PubMed: https://pubmed.ncbi.nlm.nih.gov/19185645/

27. Torsney E, Pirianov G, Charolidi N, Shoreim A, Gaze D, et al Elevation of Plasma High-Density Lipoproteins Inhibits Development of Experimental Abdominal Aortic Aneurysms. Arteriosclerosis, Thrombosis, and Vascular Biology. 2012; 32: 2678-2686. PubMed: https://pubmed.ncbi.nlm.nih.gov/23023368/ 